\title{
Resistance of spring bread wheat lines developed by ARISER to abiotic stress and rust pathogens
}

\author{
Baranova O.A. ${ }^{1 *}$, Sibikeev S.N. ${ }^{2}$, Druzhin A.E. ${ }^{2}$, Gultyaeva E.I. ${ }^{1}$ \\ ${ }^{1}$ All-Russian Institute of Plant Protection (FSBSI VIZR), St. Petersburg-Pushkin, Russia \\ ${ }^{2}$ Agricultural Research Institute of the South-East Region (FSGFSI ARISER), Saratov, Russia \\ * email: baranova_oa@mail.ru
}

In this work, we analyzed 189 spring bread wheat introgression lines developed by ARISER with alien genetic material from different species of the genus Triticum and Aegilops, Agropyron elongatum, Ag. intermedium, Secale cereale, triticale variety Satu. The results of field evaluation in the seasons 2016-2019 characterize the introgression lines taken in the analysis as highly resistant to Saratov populations of leaf rust (Puccinia triticina Erikss.) and stem rust ( $P$. graminis f. sp. tritici). However, out of 158 lines resistant to $P$. graminis in 2016, 65 lines (41.1\%) were resistant in 2020 due to loss of efficacy of Sr25 and Sr6Agi genes in the Volga region. Based on the results of the laboratory evaluation of juvenile resistance, 50 lines highly resistant to stem rust were identified. The resistant lines carried mainly combinations of $S r 31 / L r 26+S r 25 / L r 19$ and Sr31/Lr26+Sr6Agi/Lr6Agi genes, in one line a combination of Sr31/Lr26+Sr25/Lr19+Sr28+Lr10, in one line Sr25/Lr19+Sr22+Lr10 and in one line $S r 31 / L r 26+S r 57 / L r 34+L r 1+L r 3$ genes were identified. The analysis of introgression lines revealed their high genetic diversity for resistance to leaf rust. The following effective $L r$ - genes combinations were identified: $L r 19+L r 26, L r 10+L r 19+L r 26$, Lr19+Lr37, Lr19+Lr26+Lr39, Lr19+Lr6Agi, Lr19+Lr37+Lr6Agi, Lr10+Lr26+Lr6Agi, Lr10+Lr19+Lr39+Lr6Agi, Lr10+Lr39+Lr6Agi and $L r 1+L r 3+L r 34$. The agronomic performance of resistant lines was determined for a set of economically valuable traits such as grain productivity, lodging resistance and flour and bread making quality parameters. The 2018 - 2020 growing seasons were drought seasons, with 2018 and 2019 as hard droughty. Thus, resistant to complex diseases lines were also evaluated for drought tolerance. The wheat lines \# $14=\mathrm{L} 375=\mathrm{L} 505$ /3/Croc/Ae. squ(205)/Weav/4/L505/5/L505 (Lr19/Sr25+Lr26/Sr31+Lr39) and \# $20=$ $=\mathrm{L} 496 / 16$ L505*2/Proc//Bel (Lr6Agi/Sr6Agi+Lr26/Sr31+Sr28+Lr10), exceeding the standard variety Favorit for grain productivity and bread making quality and combined the drought tolerance with high resistance to stem and leaf rusts have been selected. Acknowledgements: The work was supported by RFBR grant No. 18-016-00170 a. 